\title{
QUARTZ SINGLE GRAIN OSL SENSITIVITY DISTRIBUTIONS: IMPLICATIONS FOR MULTIPLE GRAIN SINGLE ALIQUOT DATING
}

\author{
EDWARD J. RHODES \\ CRC LEME \\ Research Schools of Earth Sciences and Pacific and Asian Studies \\ The Australian National University \\ Canberra, ACT 0200, Australia
}

Received 19 September 2006

Accepted 6 November 2006

\begin{abstract}
A simple probabilistic numerical model was used to construct synthetic $\mathrm{D}_{\mathrm{e}}$ distributions by combining the simulated OSL from grains of different sensitivities and with various simulated $D_{e}$ values. This is useful for exploring the complex patterns generated by summing the signals from many grains in real, multiple grain OSL dating scenarios, and demonstrates that the form of the sensitivity distribution has a significant effect on the identification of mixed dose populations in multiple grain single aliquot methods. I describe the simple numerical model and its implementation. I present measured quartz single grain OSL sensitivity distributions for an aeolian dune sample from the Simpson Desert, Northern Territory, Australia, and from a suite of 9 fluvial sediments from Namadgi National Park, Australian Capital Territory, Australia, to illustrate effects that result from natural differences. Minimum age model determinations of synthetic $D_{e}$ values are used to assess the validity of dose values estimated from multiple grain single aliquot measurements.
\end{abstract}

Keywords: OSL, single grain, sensitivity distributions, numerical modelling

\section{INTRODUCTION}

Using a simple numerical model, I demonstrate that the pattern of single grain optically-stimulated luminescence (OSL) sensitivity distribution is the controlling factor in the propensity of a sample to provide clear indications of mixed dose populations. However, the model results are in some ways counter-intuitive, and subtle variations in the form of the single grain sensitivity distribution can have profound effects on the observed dose distributions in multiple grain OSL dating. The interpretation of these dose distributions is key to the correct analysis of OSL data, and also in selecting details of measurement procedures, in particular aliquot size and number.

Age determination based on conventional, multiplegrain, OSL measurements of quartz has demonstrated that the technique is capable of providing reliable age estimates for a wide range of different sedimentary environ-

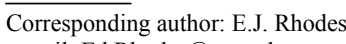

e-mail: Ed.Rhodes@anu.edu.au

ISSN 1897-1695 (online) (c) 2007 GADAM Centre,

Institute of Physics, Silesian University of Technology.

All rights reserved. ments (e.g. Smith et al., 1990; Aitken, 1998). Using the single aliquot regenerative-dose (SAR) protocol (Murray and Wintle, 2000; 2003), a high degree of precision and accuracy can be achieved (Rhodes et al., 2003; Murray and Funder, 2003). Observations of inter-aliquot variations in equivalent dose $\left(D_{e}\right)$ (Olley et al., 1998), rising $D_{e}$ values as a function of measurement time (shine plateaux or $\mathrm{D}_{\mathrm{e}}(\mathrm{t})$ plots), and for modern samples, non-zero $D_{e}$ values (Rhodes and Pownall, 1994) have all led to significant concerns over the degree of OSL signal zeroing experienced in some depositional contexts. Primarily to deal with issues of incomplete zeroing, quartz single grain OSL methods were introduced (Murray and Roberts, 1998), now routinely based on the SAR protocol (Murray and Wintle, 2000; 2003).

Despite this significant development in OSL methodology, and the introduction of purpose-built measurement equipment (Duller et al., 1999; Bøtter-Jensen et al., 2000), there has been only relatively modest uptake of single-grain OSL for routine age determination. The main reason for this is probably the significantly greater measurement and data analysis times involved when making 
single grain determinations. A major factor which contributes to this is the presence in most samples of a large proportion of grains which contribute very little luminescence signal, and therefore contribute very little useful age information. In most single grain approaches, it is not possible to avoid the measurement of these relatively insensitive grains. This extends measurement time greatly while contributing little or no useful additional information.

A second factor may be that despite expectations to the contrary, multiple grain aliquots often provide clear evidence of non-uniform dose values, despite the relatively high numbers of grains on each aliquot. In conventional, multiple grain OSL measurements, grains are adhered to the central part of a $1 \mathrm{~cm}$ diameter metal mounting disc using viscous silicone oil. For grains of $200 \mu \mathrm{m}$ diameter, a closely packed circular monolayer of $3 \mathrm{~mm}$ diameter will consist of around 200 grains, while a large $8 \mathrm{~mm}$ diameter aliquot may contain up to 1400 grains. For finer grain sizes, significantly larger grain numbers will be included within aliquots of equivalent diameter.

One relatively popular approach for the dating of contexts in which incomplete OSL signal zeroing is expected, such as high-energy fluvial deposits, uses small multiple grain aliquots, each comprising a low number of grains. For example, Olley et al. (1998) made aliquots of 60 to 80 grains to study the OSL signal zeroing in modern fluvial and aeolian sediments from Australia. This method is sometimes viewed as somehow intermediate between the measurement of single grains and conventional aliquots.

Both measurement of small aliquots (Olley et al., 1998) and numerical modelling (Wallinga, 2002) confirm that the signature of incomplete zeroing can be identified from the apparent dose distributions using multiple grain aliquots. Wallinga determined from modelling that the sensitivity distribution of constituent grains, original dose distribution and aliquot size (grain number) all affected the degree to which incomplete zeroing could be identified.

The aims of this paper are to help address the following questions:

- why can large aliquots comprising several hundred grains provide clear evidence of non-uniform dose distributions?

- for an idealized sample based on measured OSL characteristics, what is the likelihood of determining the correct dose value in the presence of increasing amounts of "contamination" by grains with a different dose value?

- is there an optimum multiple grain aliquot size to best detect mixed dose populations, and to retrieve meaningful doses from the observed values?

- under what circumstances can or should outlying dose values be rejected from multiple grain single aliquot dose distributions?

- when well-defined peaks in measured dose distributions are observed, are these values meaningful in terms of sample age, or artefacts of the multi-grain procedure adopted?
In this paper I present measured single grain OSL sensitivity distributions for several Australian sediments. I have developed a simple numerical model to simulate idealized natural $\mathrm{D}_{\mathrm{e}}$ distributions for aliquots of different size (grain number) composed of dose values randomly selected from different single grain $D_{e}$ populations, for samples having different sensitivity distributions. Using simplified sensitivity distributions, based on measured values, I use this model to assess the applicability and efficacy of existing approaches, to investigate the potential of improving these approaches, and to explore the sometimes counter-intuitive results generated by the summing of complex mixtures of signals. A key finding is that although measured distributions appear to show a power law relationship between frequency and sensitivity, modelling demonstrates that samples with this relationship tend to suppress the appearance of mixed dose values in larger multigrain single aliquot measurements. This is in marked contrast to measured data sets which often show signs of mixed dose populations.

\section{EQUIVALENT DOSE DISTRIBUTIONS FROM MULTIPLE GRAIN ALIQUOTS}

Where multiple grain single aliquot OSL measurements provide a range of dose values, the dating specialist may choose to accept a dominant group of aliquots and reject outliers. This avenue is particularly attractive in cases where a low proportion of aliquots provide significantly higher apparent equivalent dose values than those provided by a dominant group which have internally consistent dose values. The effects of incomplete zeroing may be cited as the cause of the observed variation in equivalent dose value, as justification for rejection of the dose values from outlying aliquots. However, in such situations, it is rare to have additional information to support the inferred incomplete zeroing mechanism, and it is not known whether the sample still retains a degree of dose over-estimation resulting from the effects of dimmer grains on the included aliquots.

Possible alternative strategies, instead of rejecting clear equivalent dose outliers, include the complete rejection of the sample on the grounds that the result is potentially compromised by the effects of incomplete zeroing, or to place a strong caveat or warning on the estimated age. Another possibility is to use a statistical procedure to determine the dose from the full dataset, such as the minimum age model of Galbraith et al. (1999).

For many samples, measurements made using single grains provide an improved determination of the presence of mixed $D_{e}$ populations caused, for example, by incomplete signal zeroing at the time of deposition. Multiple grain aliquots potentially suffer from OSL signal averaging; this effect is described by the central limit theorem (Tijms, 2004) and is illustrated in Fig. 1. Equivalent dose distributions from measurements, each based on the summed signals of a relatively small number (e.g. 16) of similar sensitivity grains, will inevitably be close to a normal distribution, whatever the original single grain distribution. Figs 1a-1f show simulation results for a mixture of identical sensitivity grains, of which $50 \%$ have a dose of $1 \mathrm{~Gy}$, and $50 \%$ a dose of $3 \mathrm{~Gy}$. Each plot shows 

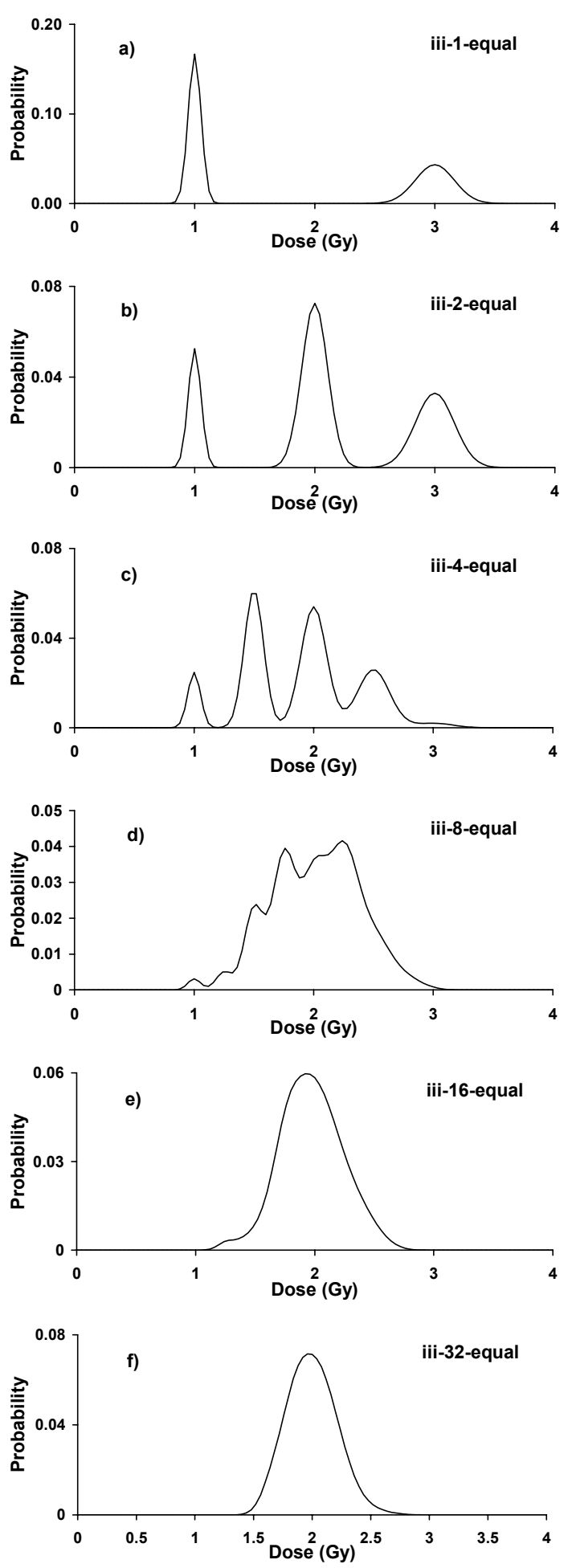

Fig. 1. Probability density function (pdf) plots of six simulated $D_{e}$ distributions each based on 96 aliquots, comprising different numbers of equal sensitivity grains randomly selected from a single grain dose population composed of 50\% $1 \mathrm{~Gy}$ and $50 \% 3 \mathrm{~Gy}$ (mixture iii in Table 2). From the top the number of grains is $1,2,4,8,16$ and 32 respectively. Codes in top right of each plot indicate dose mixture (Table 2), grain number, and sensitivity distribution. Each simulated OSL measurement has been given an uncertainty of $5 \%$. These plots clearly illustrate the averaging effects of measurements made on multiple grains of equal sensitivity (e and f), and the complex distributions which result from low numbers of grains ( $b$ and $c$ ). Fig. a also illustrates the way in which a uniform fractional uncertainty (5\%) widens and lowers the peak centred at $3 \mathrm{~Gy}$ in comparison to that at $1 \mathrm{~Gy}$. the resultant $D_{e}$ probability distribution function (pdf) for aliquots comprising different numbers of grains; simulations are for aliquots of $1,2,4,8,16$ and 32 grains per aliquot. For each plot, 96 aliquots have been simulated using the numerical model described in detail below. In order to plot the results, each simulated $D_{e}$ value has a uniform 5\% uncertainty associated with it. Note that plotting a pdf causes a reduction in height of higher $D_{e}$ peaks; equal probabilities are represented by equal areas, and a uniform fractional error (in this case 5\%) causes a widened, and therefore lowered, distribution of higher $\mathrm{D}_{\mathrm{e}}$ value peaks relative to lower peaks, as in Fig. 1a.

In the case of a sample displaying equal sensitivity grains, the correct $\mathrm{D}_{\mathrm{e}}$ distribution can only be recovered in a simple fashion using the measurement of individual grains (Fig. 1a). Note, however, that each measurement is of equal statistical value. If any individual measurement can provide a significant $D_{e}$ determination, then a good assessment of the correct $\mathrm{D}_{\mathrm{e}}$ distribution can be made relatively rapidly by measuring around 20 to 50 grains, more for complex distributions with many components.

Observations of aliquots of approximately equal mass from different samples, and comparisons between different aliquots of a single sample, clearly illustrate that the OSL light intensity that results from a uniform irradiation treatment, the OSL sensitivity, varies widely (Rhodes, 1990). As many samples of interest in luminescence dating represent natural mixtures of grains from different geological sources, samples with uniform sensitivity, displaying behaviour similar to that illustrated in Fig. 1, should not be expected. In fact, samples from monolithological primary quartz sources still display significant variation in sensitivity, and Adamiec (2000a, b) has demonstrated that even different pieces of a single natural quartz crystal display very different TL and OSL characteristics and sensitivities. Measurements of single grains of quartz have demonstrated that much of the light in multiple-grain aliquots comes from a small proportion of grains (e.g. Murray and Wintle, 2002), with variations observed in the OSL sensitivity distributions from different samples.

In order to explore realistic behaviour using the numerical simulations described below, it is important that the inputs to the model are based on realistic luminescence characteristics. Therefore, the measured OSL sensitivity distributions from several samples have been used to construct the modelled sensitivity distributions.

\section{SAMPLE LOCATIONS}

The OSL sensitivity distributions of single quartz grains from 10 samples have been measured and are presented below, in each case using the $180-210 \mu \mathrm{m}$ fraction. The first is an aeolian sand sample collected from a longitudinal dune within the Simpson Desert just north of the Finke River on the property of New Crown, east of Finke, Northern Territory, Australia, at $25^{\circ} 35^{\prime} \mathrm{S}$ $134^{\circ} 57^{\prime} \mathrm{E}$. The sample was collected with a hollow auger truck-mounted drill rig from a depth of $2.9 \mathrm{~m}$, using a 20 cm length steel cylinder, with sediment from both ends discarded in the laboratory. 
The remaining nine sediment samples come from different depths in a single profile comprising a series of silty overbank flood deposits. The samples were collected from overbank sediments exposed in a bank profile of the Cotter River, Namadgi National Park, ACT Australia at $35^{\circ} 37^{\prime} \mathrm{S} 148^{\circ} 50^{\prime} \mathrm{E}$. Forty-eight single grains were measured for each of the 9 samples as part of an on-going study of environmental response to bush fires. A composite sensitivity distribution has been created by combining the results from all 432 grains.

\section{PREPARATION AND OSL MEASUREMENT}

Quartz grains were separated following a routine preparation procedure under $\operatorname{dim} 588 \mathrm{~nm}$ laboratory lighting, involving sieving, concentrated HF treatment constantly agitated for 1 hour with $\mathrm{HCl}$ present, removal of heavy minerals using a solution of sodium polytungstate with a density of $2.68 \mathrm{~g} \mathrm{~cm}^{-3}$, and re-sieving to remove grain fragments attacked by the acid treatment. Single grains were mounted on $1 \mathrm{~cm}$ diameter stainless steel discs with a small spot of viscous silicone oil.

OSL measurements were made in a Risø TL-DA-15 mini-sys automated luminescence reader, fitted with 28 Nichia NSPB500S blue LEDs providing $35 \mathrm{~mW}$ stimulation centred at $470 \mathrm{~nm}$, with detection through a $7.5 \mathrm{~mm}$ coated Hoya U340 filter using an EMI QA9235 photomultiplier. OSL measurements were performed at $125^{\circ} \mathrm{C}$, and were preceded by IRSL measurements at $60^{\circ} \mathrm{C}$. Sensitivity measurements were preceded by a $10 \mathrm{~s}$ heat at $220^{\circ} \mathrm{C}$ for sample $\mathrm{K} 0207$ and $200^{\circ} \mathrm{C}$ for the Cotter River sample suite, and represent the net initial OSL response to a 6 Gy test dose following the measurement of the natural OSL.

\section{MEASURED SINGLE GRAIN SENSITIVITY DISTRIBUTIONS}

The OSL sensitivity distribution observed for 96 quartz grains of aeolian dune sample K0207 is shown in
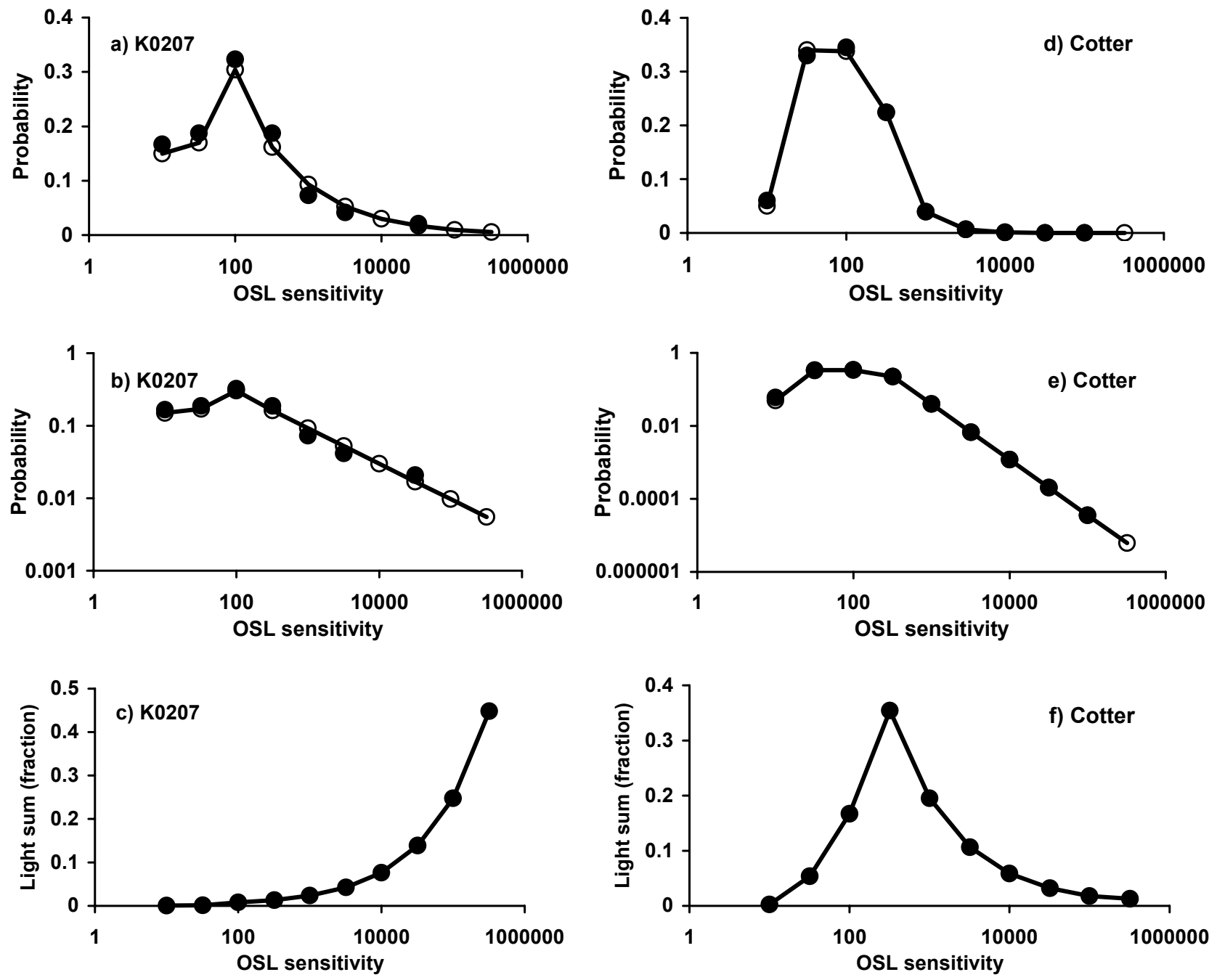

Fig. 2. Measured single grain OSL sensitivity frequencies are shown as solid symbols; a and b show data for Simpson Desert aeolian sample K0207, and $d$ and e show combined data from 9 fluvial samples from the Cotter River catchment, ACT. Open symbols show values used as input for numeri$\mathrm{cal}$ model simulations. Figs $b$ and e illustrate the striking power law relationship between probability and sensitivity. This relationship has been used to extrapolate model input values at higher sensitivities. Also shown are the contributions to the overall OSL light sum of different sensitivity classes for K0207 (c) and the combined Cotter River samples (f), illustrating the very different sources of OSL signal between the two groups. 
Table 1. Characteristics of the high sensitivity power law relationships with frequency for measured single grain distributions. All samples except X090 are from Australia, letter codes are for states and territories: NT=Northern Territory, ACT=Australian Capital Territory, SA=South Australia, WA=Western Australia, NSW=New South Wales. Sample codes, numbers of measured grains, location, depositional environment and the fitted mathematical relationship between the higher sensitivities and probability are shown. All samples were measured on individual stainless steel discs except for X090 which was measured using a Risø single grain laser attachment.

\begin{tabular}{lclll}
\hline Sample code & Grain no. & Location & $\begin{array}{l}\text { Depositional environ- } \\
\text { ment }\end{array}$ & Relationship \\
\hline K0207 & & & aeolian & $y=2.73 x^{-0.49}$ \\
Cotter River & 96 & Simpson Desert, NT & fluvial & $y=1460 x^{-1.52}$ \\
K0010 & 432 & Namadgi, ACT & aeolian & $y=3.93 x^{-0.57}$ \\
K0329 & 48 & Strzelecki Desert, SA & aeolian & $y=8.22 x^{-0.65}$ \\
CE1 & 48 & Russell Hill, ACT & annealed & $y=203 x^{-0.83}$ \\
Genoa River & 336 & NSW & fluvial & $y=238 x^{-0.71}$ \\
X090 & 1600 & Old Scatness Broch, UK & hearth & $y=240 x^{-1.30}$ \\
\hline
\end{tabular}

Fig. 2a. The solid symbols represent measured OSL sensitivity data, and the open symbols represent the values used as input for numerical modelling. The same data are shown in Fig. 2b, plotted with a logarithmic y-axis. The single grain sensitivities have been plotted as the fraction of measured grains within half order of magnitude steps. That is, the number of grains with test-dose responses of up to $10,32,100,320,1000,3200,10000,32000$, 100000 and $320000 \mathrm{cps}$ (for a 6 Gy test dose) are plotted. The values are plotted at the highest sensitivity value within each class, as this better approximates the dominant effects of brighter grains.

Fig. 2c shows the fractional contribution to the overall OSL response of each sensitivity class. For sample K0207, the highest light sum value is at 320,000 cps. Clearly, in this sample, the brighter grains contribute a much greater fraction of the total OSL signals than do the dimmer grains.

In contrast, the combined sensitivity distribution constructed from 9 fluvial samples from the Cotter river catchment, Namadgi National Park, ACT, is shown in Figs 2d and 2e. This sample suite is characterised by a higher proportion of grains having lower sensitivities, and a very different relationship between frequency and sensitivity for the higher sensitivity grains. This is clearly seen by comparing Figs $\mathbf{2 b}$ and $\mathbf{2 e}$ (note the different y-axis scales). In both samples, the more sensitive grains form an approximately power law relationship between frequency (probability) and sensitivity. This approximate power law relationship forms a straight line in both of the two log-log plots (Figs 2b and 2e). However, note that the slope of the high sensitivity power law relationship for the Cotter River samples (Fig. 2e) is much greater than that for sample K0207 (Fig. 2b). In other words, brighter grains are proportionately rarer for the fluvial, Cotter River samples than for the aeolian sample, K0207.

The high sensitivity power law relationships with frequency have been quantified for these two sample suites by fitting the data points above the sensitivity peak, as shown in Table 1. A similar approximately power law relationship between frequency and sensitivity has been observed for single grain distributions from many other samples, both by measuring the grains mounted on individual discs and using a Risø single grain laser attachment. Relationships for several other samples are also shown in Table 1.
The physical mechanism responsible for this power law relationship is not understood at present. In simple terms, for each order of magnitude change in OSL sensitivity, there is a constant change in the probability of finding a grain with that sensitivity, independent of the initial sensitivity. For exponents smaller than -1.0 (e.g. 1.30 for sample X090, or -1.52 for the Cotter River sample suite), the light sum has a peak at or around the dominant sensitivity class, as for the Cotter river suite, shown in Figs 2d, 2e and 2f. For samples with exponents greater than -1.0 (e.g. -0.49 for sample K0207, or -0.57 for sample K0010), the light sum will be dominated by the brightest sensitivity class, as illustrated by Figs 2a-2c for sample K0207.

It is clear from the small survey of samples included in Table 1 that for the 6 samples or sample suites from Australia, 5 have exponent values greater than -1.0, and therefore have fractional light sum distributions as a function of sensitivity more like that of sample K0207 (Fig. 2c) than that of the Cotter River suite (Fig. 2f). It is how these different sensitivity patterns impact $D_{e}$ distributions measured using the summed OSL signals from multiple grains on individual aliquots that the numerical model described below was designed to assess.

\section{NUMERICAL SIMULATIONS OF MULTIPLE GRAIN ALIQUOTS}

A simple numerical model was constructed in order to generate synthetic $\mathrm{D}_{\mathrm{e}}$ distributions based on varying numbers $(n)$ of grains. Simulations were performed using $n=1,10,100$ and 500 grains. In the simulations presented here, each grain was allocated one of two possible dose values, either 1 or $3 \mathrm{~Gy}$. This allocation was made using random numbers, with a pre-determined probability for each dose value. Four different dose mixtures were simulated, namely i) $95 \% 1 \mathrm{~Gy}, 5 \% 3 \mathrm{~Gy}$, ii) $70 \% 1 \mathrm{~Gy}$, $30 \% 3 \mathrm{~Gy}$, iii) $50 \% 1 \mathrm{~Gy}, 50 \% 3 \mathrm{~Gy}$, and iv) $10 \% 1 \mathrm{~Gy}$ and $90 \% 3$ Gy (see Table 2). Each grain was also allocated a sensitivity, independently randomly selected using the probabilities shown as open symbols in Figs 2a and $\mathbf{2 b}$ for sample K0207, and Figs 2d and 2e for the Cotter River sample suite. Note that for the modelled sensitivity values, the apparent power law relationship was extrapolated to higher sensitivities (to $1 \times 10^{8}$ ), corresponding to very low probabilities, or very rare grains. 
Table 2. Proportions of grains in each of the four different simulated dose mixtures used in the numerical modelling.

\begin{tabular}{cccc}
\hline $\begin{array}{c}\text { Mixture } \\
\text { number }\end{array}$ & $\begin{array}{c}\text { Proportion of grains } \\
\text { with 1 Gy dose }\end{array}$ & $\begin{array}{c}\text { Proportion of grains } \\
\text { with 3 Gy dose }\end{array}$ & Total \\
\hline i & 0.95 & 0.05 & 1.00 \\
ii & 0.70 & 0.30 & 1.00 \\
iii & 0.50 & 0.50 & 1.00 \\
iv & 0.10 & 0.90 & 1.00 \\
\hline
\end{tabular}

A synthetic natural OSL signal was simulated using the product of the sensitivity and the allocated dose value for each grain. The OSL response of each grain to a (notional) 1 Gy test dose was provided simply by the sensitivity value allocated to each grain. To simulate the total natural OSL signal of each synthetic aliquot, the sum of all the synthetic natural OSL signals for each of the $n$ simulated grains was taken, and to simulate the test dose response for each aliquot, the synthetic test dose OSL value for each grain was summed. To determine the synthetic $D_{e}$ value for each aliquot, the synthetic natural OSL signal was divided by the synthetic test dose response. This is analogous to a situation in which grains do not change in sensitivity during a SAR measurement cycle, and all grow linearly over the simulated dose range, with zero intercept.

Note that this simulation provides synthetic single grains of different sensitivities (and therefore different natural OSL signals), but with dose values of exactly 1 or 3 Gy. No measurement error is simulated, even for the low sensitivity grains. In summary, the simple simulations described here provide idealized grain behaviour, with no changes in sensitivity, zero intercept, linear growth, and most importantly no measurement error even for low sensitivity grains. The only cause of variation between different aliquots in the same simulation run is caused by the probabilistic variation in $\mathrm{D}_{\mathrm{e}}$ and sensitivity
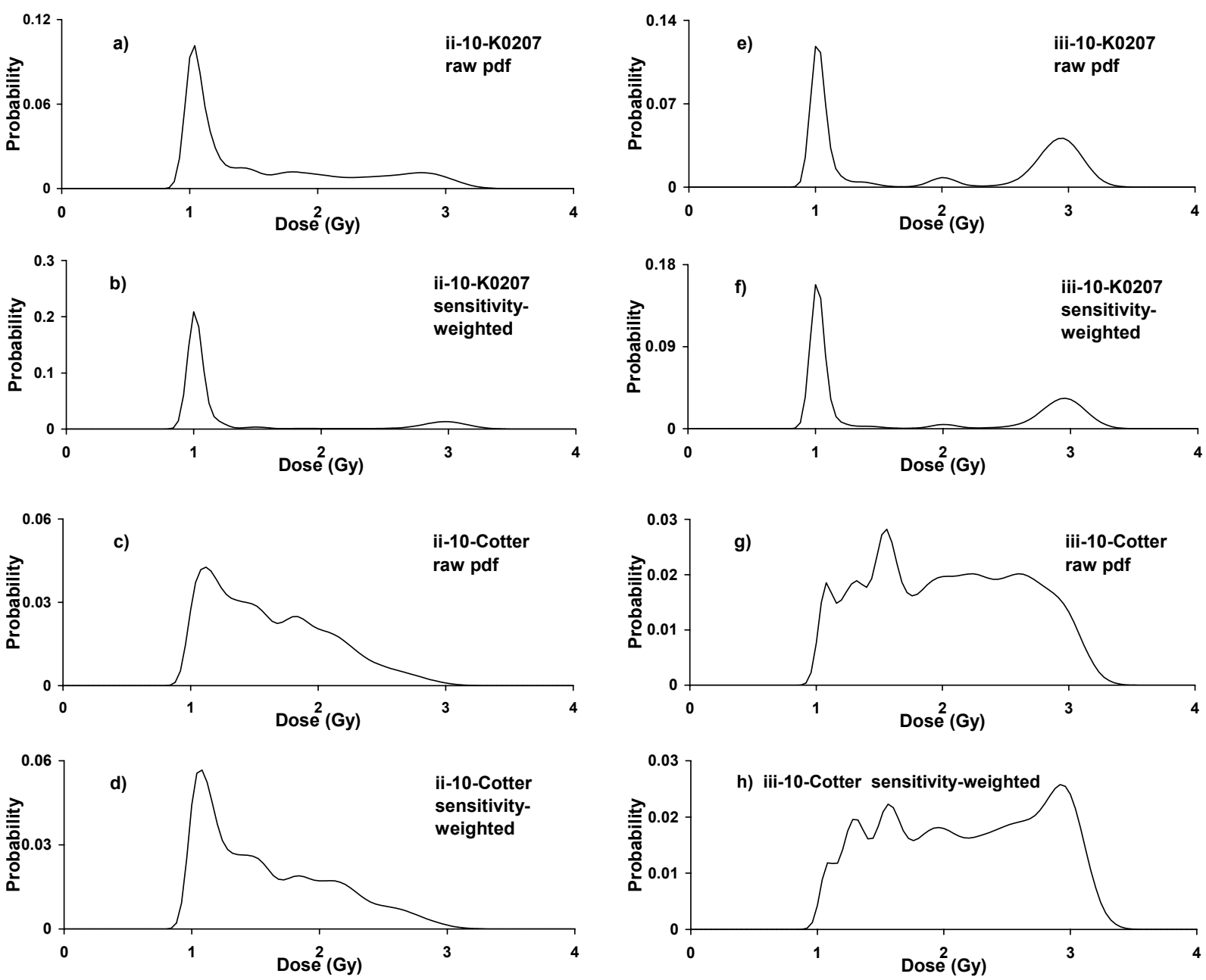

Fig. 3. Probability distribution function (pdf) plots of simulated 10 grain $D_{e}$ distributions for samples K0207 above (a and b, e and f) and Cotter River below ( $c$ and $d, g$ and $h$ ). Each plot represents the sum of 96 simulated aliquots each comprising 10 grains. The plots on the left represent an underlying $D_{e}$ distribution comprising mixture ii (1 Gy 70\%, 3 Gy 30\%; Table 2), and those on the right were constructed using mixture iii (1 Gy $50 \%$, $3 \mathrm{~Gy}$ $50 \%)$. The upper plot of each pair, that is Fig. $3 a, c$, e and g, represents the raw pdf plot, while in the lower plot of each pair (b, $d$, $f$ and $h)$ each of the 96 individual Gaussians was multiplied by the sensitivity of that simulated aliquot, to provide a sensitivity-weighted pdf. Codes in the top right of each plot indicate dose mixture (Table 2), grain number, and sensitivity distribution. Some indication of the form of the underlying $D_{e}$ distribution is provided by the K0207 sensitivity-weighted pdf plots ( $b$ and $f$ ), while in all other cases there is unambiguous indication of a non-uniform underlying $D_{e}$ distribution. 

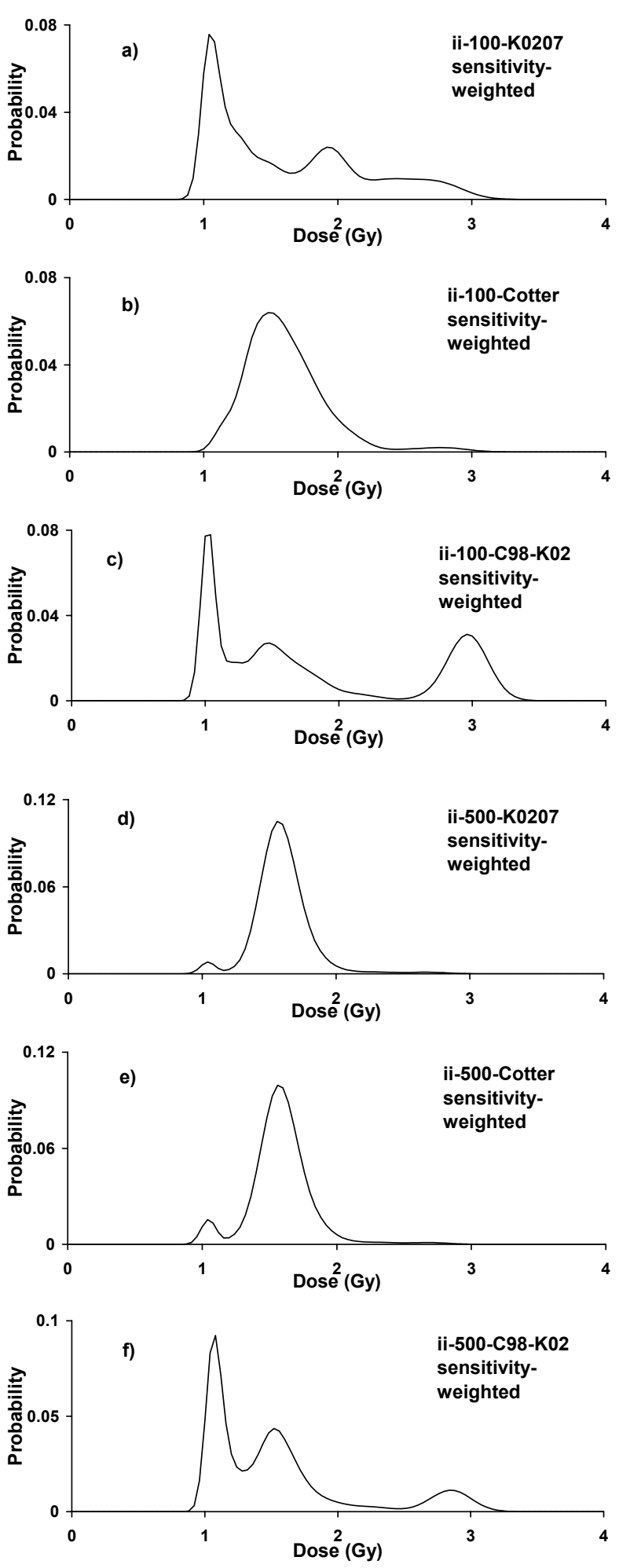

Fig. 4. Sensitivity-weighted pdf plots for simulated 100 (a, b, c) and 500 grain (d, e, f) aliquots, using dose mixture ii $11 \mathrm{~Gy} 70 \%$, 3 Gy $30 \%$; Table 2). Each plot represents the sum of 384 simulated aliquots using the single grain sensitivity distribution for sample $K 0207(a, d)$, the Cotter River sample suite $(b, e)$ and a composite distribution comprising 98\% Cotter River and 2\% K0207 (c, f). Codes in the top right of each plot indicate dose mixture (Table 2), grain number, and sensitivity distribution. Using 500 grains, the composite simulation (f) appears to provide a clearer representation of the underlying $D_{e}$ distribution than do the K0207 or Cotter River simulations, though with an additional intermediate dose value observed, while at 100 grains the K0207 simulation also displays multiple dose peaks. of the contributing simulated grains.

In order to plot the data in pdf format, which provides a useful visualisation of the relative importance of different dose components, a uniform 5\% uncertainty has been assumed for all aliquots.

\section{SIMULATED $\mathrm{D}_{\mathrm{e}}$ DISTRIBUTIONS}

Equivalent dose $\left(\mathrm{D}_{\mathrm{e}}\right)$ distributions based on 384 synthetic aliquots have been simulated for four different single grain dose mixtures (detailed above, see Table 2), using four aliquot sizes (see above), for the two sensitivity distributions shown in Fig. 2.

The simulations for $n=1$ (single grains) provide single aliquot $\mathrm{D}_{\mathrm{e}}$ values of either 1 or $3 \mathrm{~Gy}$, approximately in the proportions for the selected dose mixture. The proportions of grains at each dose value do not match the proportions in Table 2 exactly, owing to the random selection of dose value for each grain.

For the simulations where $n=10$, that is each simulated aliquot consists of 10 grains, the apparent $D_{e}$ value of individual aliquots can range between 1 and $3 \mathrm{~Gy}$, depending on the number of grains with each dose value, and also on their sensitivities. In order to help visualise the resultant $\mathrm{D}_{\mathrm{e}}$ distributions, pdf plots are presented in Fig. 3, for two dose mixtures. For the simulations based on sample K0207, peaks in the pdf plots close to 1 and 3 Gy are observed (Figs 3a and 3e). These peaks are primarily caused by the presence of a significant number of aliquots which have a single bright grain dominating the signal, and hence provide apparent $D_{e}$ values close to 1 or 3 Gy. A smaller contribution to these peaks is from aliquots with two or more brighter grains coincidentally having the same dose value of either 1 or $3 \mathrm{~Gy}$.

These simulations clearly illustrate how variations in the sensitivities of the constituent grains for different aliquots provide a means to detect non-uniform dose distributions; these samples are not subject to the same degree of signal averaging as for the uniform grain sensitivity simulations shown in Fig. 1.

As described above, it is the domination of the OSL signal by one or a small number of bright grains which allows hints of the underlying single grain distribution to be detected. One means to exaggerate this effect is to amplify the signals from brighter aliquots. This may be done by multiplying the raw pdf for each aliquot by its sensitivity before summing as a combined multiple aliquot pdf, to provide a sensitivity-weighted pdf. This has been done for the $n=10$ simulations; resultant plots are shown in Fig. 3 (b, d, $\mathbf{f}$ and $\mathbf{h}$ ). The sensitivity-weighted pdf plots are envisaged purely as a means to assist visualisation, rather than in attempting to recover dose values from $\mathrm{D}_{\mathrm{e}}$ distributions.

For the $n=10$ simulations, those based on the sensitivity distribution of aeolian sample K0207 from the Simpson Desert seem more useful than those for the fluvial Cotter River sample suite in terms of detecting a non-uniform dose distribution, and for providing indications of the dose values and proportions.

From the $n=100$ simulations, the Cotter River sample suite appears to provide almost no indication of a mixed dose (Fig. 4b) for the 70\% 1 Gy, 30\% 3Gy mix- 
ture (mixture ii), while the aeolian sample K0207 has a significant peak close to $1 \mathrm{~Gy}$, a secondary peak at around 2 Gy and values extending up to 3 Gy in the sensitivity-weighted pdf (Fig. 4a). For the same dose mixture (ii), the $n=500$ grain simulations of both samples provide sensitivity-weighted pdf plots with a dominant peak at around $1.6 \mathrm{~Gy}$, and a tiny secondary peak just above $1 \mathrm{~Gy}$.

\section{DISCUSSION}

For non-uniform OSL sensitivity distributions, the summing of signals from multiple grains in the determination of equivalent dose values does not entirely mask the presence of mixed dose populations. This is because occasional high sensitivity grains on some aliquots are able to dominate the total measured OSL signal for that aliquot. This effect is clearly illustrated by the simulations shown in Figs 3b, 3f and $\mathbf{4 a}$.

Samples with different single grain OSL sensitivity distributions have varying degrees to which a similar underlying dose mixture can be determined by the measurement of multiple grain aliquots. This effect is clearly illustrated by comparing Figs 3b (K0207) and 3d (Cotter), or Figs 4a (K0207) and $\mathbf{4 b}$ (Cotter).

The propensity of the sample to represent its underlying single grain $\mathrm{D}_{\mathrm{e}}$ distribution is controlled primarily by the proportion of bright grains present, and their ability to dominate the OSL output of an individual aliquot. If there are too many bright grains present, the averaging effects of multiple bright grains on each aliquot reduce the sample's propensity to be a faithful recorder of the underlying single grain $\mathrm{D}_{\mathrm{e}}$ distribution.

These simulations suggest that above a certain aliquot
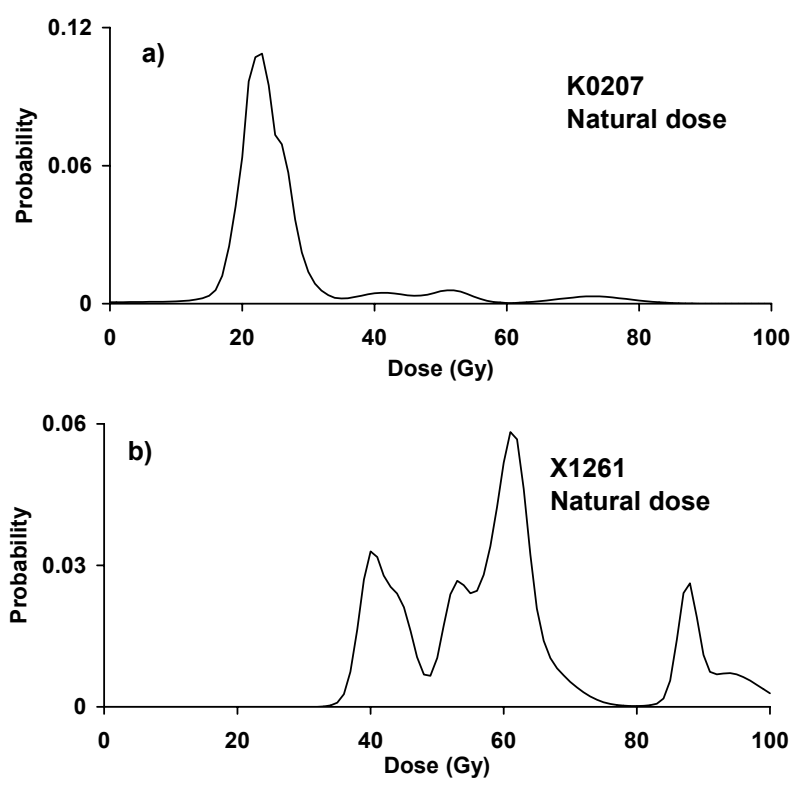

Fig. 5. Measured pdf plots of natural equivalent dose for samples a) K0207 (Simpson Desert) and b) X1261, a sandy glacial outwash sediment from the Scilly Isles, UK (see text for further details). Both distributions show clear signs of mixed dose populations. Approximate aliquot sizes were $n=200$ for K0207 and $n=800$ for X1261. size, or value of $n$ grains, mixed dose distributions are unlikely to be observed. For example, based on the simulations for the Cotter River fluvial sample suite, for dose mixture ii, the presence of a mixed dose is clear at $n=10$ (Figs 3c and 3d), but indicated only by a slightly widened single peak for $n=100$ (Fig. 4b). For the more sensitive aeolian sample K0207 and dose mixture ii, simulations show clear indication of mixed dose values at $n=10$ (Figs 3a and 3b) and at $n=100$ (Fig. 4a), but little indication at $n=500$ (Fig. 4d).

Equivalent dose measurements performed during the OSL dating of sample K0207 using aliquots of approximately 200 grains each are shown in Fig. 5a, not sensitivity-weighted. Of the 24 aliquots shown in this plot, 21 form a single peak at about 24 Gy, while 3 have significantly higher dose values (41, 51 and 73 Gy). This displays a range of relative dose values similar to that used in the simulations described here, that is a factor of about 3 , though the degree of differentiation of these values appears clearer than in the simulations for $n=100$ for sample K0207 (Fig. 4a).

Sample X1261 (Fig. 5b) was collected within sandy glacifluvial outwash deposit located at The Battery, Tresco, Isles of Scilly, UK, at $49^{\circ} 58^{\prime} \mathrm{N} 006^{\circ} 21^{\prime} \mathrm{W}$. Twelve aliquots were measured, each comprising approximately 800 grains of $180-250 \mu \mathrm{m}$, providing the dose distribution shown in Fig. 5b. Three distinct peaks in dose value are observed, with shoulders buttressing two. Without specific information concerning the single grain dose distribution for this sample, it is not possible to be definitive in the interpretation of these measured values, though it seems likely that the strong grouping of the dose values is significant. However, the average OSL sensitivity of this sample is very much lower than that of sample K0207, at less than $1 \%$, and lower than that of the Cotter River sample suite. If the single grain sensitivity distribution of this sample also displays a power law relationship between the probability and sensitivity for the higher values, then the low average sensitivity constrains the exponent to be significantly less than -1.00 . Simulations based on the Cotter River sample suite, and also of an entirely synthetic distribution with an exponent of -2.00 , do not display discrete dose values for aliquots of 500 grains or above for the dose mixtures listed in Table 2. From the variation of dose shown in Fig. 5b, and from the measured average sensitivity, we can be sure that this sample does not have a "regular" sensitivity distribution, that is, a distribution with a simple power law relationship between frequency and sensitivity for the higher sensitivity grains.

In order better to reproduce the behaviour displayed by sample X1261, and also by many other samples, an alternative synthetic sensitivity distribution was created by the addition of $98 \%$ grains having the distribution of the dim, fluvial sample suite from the Cotter River (Figs 2d and 2e), with $2 \%$ of grains having the bright, aeolian simulated distribution of K0207 (Figs 2a and b). The resulting sensitivity distribution is shown in Fig. 6a and 6b, along with that of the Cotter River sample suite. Note that this Figure shows sensitivity values extending to a higher class on the x-axis than does Fig. 2. It is only at these high sensitivity values that significant departure 

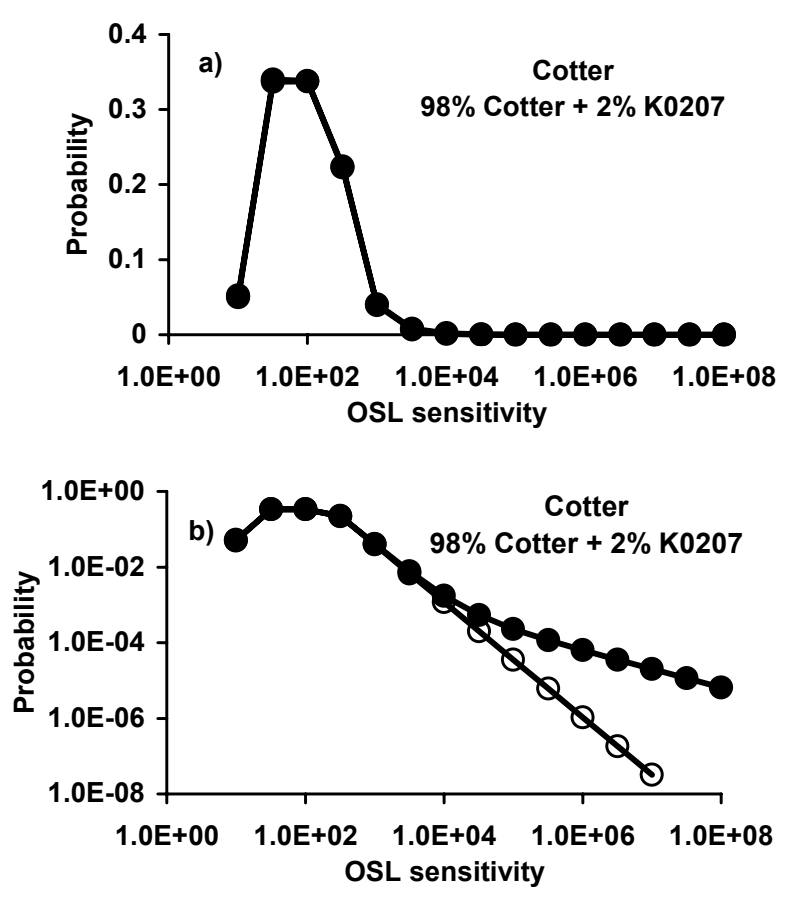

Fig. 6. Model input sensitivity distributions for the Cotter River sample suite (open symbols), and for a composite distribution comprising $98 \%$ Cotter River suite and 2\% the K0207 model input distribution (solid symbols). Note that in this Figure, the OSL sensitivity axis extends two orders of magnitude higher than those of Fig. 2.

between the two distributions occurs, and these few grains are responsible for the significant differences in modelled behaviour. Fig. 4 presents results of the numerical simulations for this mixture for dose mixture ii (Table 2), for 100 grains (Fig. 4c) and 500 grains (Fig. 4f). These results may be compared to those based on the Cotter River suite alone (Figs $4 \mathbf{b}$ and 4d). For the composite distribution, the addition of just $2 \%$ of grains from a different distribution radically alters the "visibility" of the underlying dose distribution (dose mixture ii).

The observation that a simulated distribution with a non-power law frequency-sensitivity relationship better matches the behaviour of natural samples produces a sobering conclusion. In each of the sensitivity classes simulated here, only very small fractional differences are registered between the Cotter River suite distribution and the composite distribution, except for the rare, high sensitivity classes. If 1000 grains from each had been measured to form sensitivity distributions, there would be less than a difference of one grain in any class between the Cotter River and the composite distribution. In other words, it would be extremely difficult to distinguish between the two distributions shown in Fig. 6 on the basis of single grain sensitivity measurements, yet the response of multi-grain aliquots is radically different (Fig. 4). I conclude that it is unlikely that single grain sensitivity determinations will be sufficient to predict multi-grain aliquot distributions.

\section{DOSE DETERMINATION BASED ON THE MINIMUM AGE MODEL}

These simulations demonstrate that multiple grain single aliquot measurements of real samples have the potential to reveal mixed dose distributions for their constituent grains. In simple cases such as only two contributions to the dose mixture (as simulated here), or for situations in which the only possible sources of mixed dose values are in the same sense (i.e. either greater than or less than the primary dose value), there is the potential to use methods such as the minimum age model (Galbraith et al., 1999) to isolate a meaningful dose value.

Multi-grain single aliquot dose distributions based on many aliquots may show clear peaks close to the underlying dose values (such as those shown in Figs 3 and $\mathbf{4}$ which are based on the sum of 384 simulated aliquots), but smaller subsets of aliquots may not. Practical OSL dating protocols are based on far fewer aliquots, so to assess the severity of this effect, the equivalent dose values from subsets of 12 simulated aliquots were analysed

Table 3. Equivalent dose determination from simulated OSL distributions using the minimum age model. All values shown are in Gy with associated 1 sigma uncertainties. Data are shown for 3 simulations, all using dose mixture ii (70\% $1 \mathrm{~Gy}, 30 \% 3 \mathrm{~Gy})$ for $n=10$ for the $\mathrm{K} 0207$ distribution, for $n=10$ for the Cotter River suite distribution and for $n=$ 500 for a composite distribution comprising 98\% Cotter River and $2 \%$ K0207 (see text for details). Analyses were conducted on 12 mutually exclusive, randomly selected, groups of 12 simulated aliquots, and for the same 144 aliquots analysed as a single group. Also shown are the analyses of the full 384 aliquots, whose distributions are shown in Figs $3 a, 3 c$ and $\mathbf{4 f}$ respectively. NR=not resolved.

\begin{tabular}{|c|c|c|c|}
\hline $\begin{array}{l}\text { Number of } \\
\text { aliquots }\end{array}$ & $\begin{array}{c}\text { ii-10-K0207 } \\
D_{\mathrm{e}} \pm 1 \sigma \\
(\mathrm{Gy})\end{array}$ & $\begin{array}{c}\text { ii-10-Cotter } \\
D_{\mathrm{e}} \pm 1 \sigma \\
(\mathrm{Gy})\end{array}$ & $\begin{array}{c}\text { ii-500-C98-K02 } \\
D_{\mathrm{e}} \pm 1 \sigma \\
\text { (Gy) }\end{array}$ \\
\hline 12 & $1.02_{-0.02}^{+0.02}$ & $1.02_{-\mathrm{NR}}^{+0.06}$ & $1.09_{-0.08}^{+0.07}$ \\
\hline 12 & $1.05_{-\mathrm{NR}}^{+0.06}$ & $1.08_{-0.08}^{+0.07}$ & $1.07_{-N R}^{+0.04}$ \\
\hline 12 & $1.02_{-\mathrm{NR}}^{+0.04}$ & $1.07_{-0.08}^{+0.07}$ & $1.09_{-0.05}^{+0.04}$ \\
\hline 12 & $1.03_{-0.02}^{+0.03}$ & $1.02_{-\mathrm{NR}}^{+0.05}$ & $1.41_{-0.07}^{+0.06}$ \\
\hline 12 & $1.02_{-\mathrm{NR}}^{+0.03}$ & $1.14_{-0.06}^{+0.08}$ & $1.35_{-0.06}^{+0.06}$ \\
\hline 12 & $1.07_{-0.03}^{+0.05}$ & $1.13_{-0.07}^{+0.05}$ & $1.11_{-0.09}^{+0.04}$ \\
\hline 12 & $1.04_{-0.03}^{+0.03}$ & $1.03_{-N R}^{+0.05}$ & $1.07_{-\mathrm{NR}}^{+0.07}$ \\
\hline 12 & $1.05_{-0.02}^{+0.02}$ & $1.02_{-N R}^{+0.07}$ & $1.41_{-0.06}^{+0.07}$ \\
\hline 12 & $1.01_{-\mathrm{NR}}^{+0.03}$ & $1.18_{-0.08}^{+0.08}$ & $1.11_{-0.04}^{+0.03}$ \\
\hline 12 & $1.06_{-N R}^{+0.04}$ & $1.05_{-\mathrm{NR}}^{+0.05}$ & $1.26_{-0.06}^{+0.07}$ \\
\hline 12 & $1.01_{-\mathrm{NR}}^{+0.04}$ & $1.11_{-0.05}^{+0.04}$ & $1.34_{-0.07}^{+0.05}$ \\
\hline 12 & $1.02_{-N R}^{+0.03}$ & $1.02_{-N R}^{+0.04}$ & $1.30_{-0.06}^{+0.07}$ \\
\hline 144 & $1.04_{-0.01}^{+0.01}$ & $1.06_{-0.04}^{+0.02}$ & $1.10_{-0.03}^{+0.02}$ \\
\hline 384 & $1.04_{-0.01}^{+0.01}$ & $1.05_{-0.02}^{+0.01}$ & $1.13_{-0.01}^{+0.01}$ \\
\hline
\end{tabular}


using the minimum age model for three different simulated scenarios. The results are shown in Table $\mathbf{3}$ for the distributions shown in Figs 3a, 3c and Fig. 4f, which are all based on dose mixture ii and represent $n=10$ for the simulated K0207 and Cotter River suite, and $n=500$ for the composite distribution. Twelve randomly selected subsets of 12 simulated aliquots were analysed for each distribution. Also shown are the independent analysis of the same total 144 aliquots, and the analysis of the full 384 aliquots, as shown in Figs 3a, 3c and Fig. 4f. From Table 3, it is clear that for this dose mixture $(70 \%$ of grains have $1 \mathrm{~Gy}, 30 \%$ have $3 \mathrm{~Gy}$ ), most of the minimum age model analyses of groups of 12 aliquots for the $n=$ 10 simulations approach the correct value of 1.00 moderately closely, though the lower 1 sigma uncertainty is often not resolved by the fitting procedure. The simulated K0207 distribution performs better than the Cotter River suite distribution, and every analysis has a small systematic over-estimate. For the composite distribution with $n$ $=500$, the performance is significantly worse, with 6 results $(50 \%)$ returning values $25 \%$ above the correct value. The analysis of the same 144 aliquots performed as a single group improves the approach to the correct value for this distribution, returning a value of $1.10_{-0.03}^{+0.02} \mathrm{~Gy}$, but for all distributions there is no significant improvement in increasing the aliquot number to 384 .

These results highlight potential pitfalls in the application of the minimum age model to groups of multigrain single aliquot measurements, that is a systematic overestimation in equivalent dose even when relatively widely distributed dose values are encountered. This is caused by the additional averaging effects of dimmer, contributing grains, even within aliquots that have strong signals from grains with the lower dose value.

\section{CONCLUSIONS}

Multiple grain single aliquot measurements of real samples can reveal mixed dose distributions, such as those shown in Fig. 5. This effect can be numerically simulated, at least in a general sense, as illustrated in Figs 3 and 4 , and is produced by the effects of grains of different sensitivity. The questions addressed in the introduction can now be answered, as follows.

- The effects of high sensitivity grains, which in some cases are sufficiently bright to dominate the signal from an individual aliquot, allow mixed dose populations to be observed even in large multiple grain aliquots (e.g. Fig. 4f).

- Subject to the limitations imposed by the precision of constituent grains, there is good potential for determining meaningful $\mathrm{D}_{\mathrm{e}}$ values when the proportions of "contaminant" grains is low. However, application of the minimum age model may return values with systematic over-estimates of the correct dose even when the observed dose distributions suggest good separation of the components (e.g. Fig. $4 \mathbf{f}$ and Table 3 ).

- No single multi-grain aliquot size will provide optimal detection of mixed dose populations for all samples, as the optimal size is controlled by the nature of the sensitivity distribution.
- It should be expected that multi-grain measurements reveal aliquots with outlying dose values, and that the omission of these can significantly improve the veracity of the dose estimate; however, the resulting values may still represent mis-estimates of the true value (e.g. Table 3).

- Not all the peaks measured in multi-grain single aliquot dose distributions represent meaningful dose values; they can be produced by averaging effects, as illustrated by the peaks between 1.5 and 2 Gy in Figs 4a-4f.

Simulations covering a range of different sensitivity distributions demonstrate that where the modelled data include a simple power law relationship between frequency and sensitivity, aliquots of several hundred grains are not likely to show mixed dose distributions clearly. As real, observed natural dose distributions using aliquots of several hundred grains can show mixed dose values, this strongly suggests that these samples have sensitivity distributions with different characteristics. I conclude that the measurement of a small number of single grains (e.g. 48) to determine the power-law exponent value (Table 1) is not sufficient to construct a useful predictive numerical model for aliquots of different sizes, in order to find the optimal aliquot size when planning multi-grain single aliquot measurements.

\section{ACKNOWLEDGEMENTS}

The author would like to acknowledge financial support from CRC LEME as part of the History of Aridity project. I thank Martin Worthy who collected, prepared and measured the Namadgi National Park fluvial samples, and Norman Hill who assisted in the preparation of all the samples. Kat Fitzsimmons kindy provided sample K0010. Thanks to Damien Kellerher, John Chappell and Toshi Fujioka, who helped collect the Simpson Desert sample, and to Dolan Hewitt who helped in its preparation.

\section{REFERENCES}

Adamiec G, 2000a. Variations in luminescence properties of single quartz grains and their consequences for equivalent dose estimation. Radiation Measurements 32: 427-432.

Adamiec G, 2000b. Aspects of pre-dose and other luminescence phenomena in quartz absorbed dose estimation. Unpublished DPhil thesis, University of Oxford: 200pp.

Aitken MJ, 1998. Introduction to optical dating. Oxford University Press, Oxford, 267pp.

Bøtter-Jensen L, Bulur E, Duller GAT and Murray AS, 2000. Advances in luminescence instrument systems. Radiation Measurements 32 . 523-528.

Duller GAT, Bøtter-Jensen L, Murray AS and Truscutt AJ, 1999. Single grain laser luminescence (SGLL) measurements using a novel automated reader. Nuclear Instruments and Methods in Physical Research Section B 155: 506-514.

Galbraith RF, Roberts RG, Laslett GM, Yoshida H and Olley JM, 1999. Optical dating of single and multiple grains of quartz from Jinmium rock shelter, northern Australia: Part I. Experimental design and statistical models. Archaeometry 41: 339-364.

Murray AS and Funder S, 2003. Optically stimulated luminescence dating of a Danish Eemian coastal marine deposit: a test of accuracy. Quaternary Science Reviews 22: 1177-1183.

Murray AS and Roberts RG, 1998. Measurement of the equivalent dose in quartz using a regenerative-dose single-aliquot protocol. Radiation Measurements 29: 503-515. 
Murray AS and Wintle AG, 2000. Luminescence dating of quartz using an improved single-aliquot regenerative-dose protocol. Radiation Measurements 32: 57-73.

Murray AS and Wintle AG, 2002. Retrospective dose assessment: the measurement of the dose in quartz in dating and accident dosimetry. Radiation Protection Dosimetry 101: 301-308.

Murray AS and Wintle AG, 2003. The single aliquot regenerative dose protocol: potential for improvements in reliability. Radiation Measurements 37: 377-381.

Olley JM, Caitcheon GC and Murray AS, 1998. The distribution of apparent dose as determined by optically stimulated luminscence in small aliquots of fluvial quartz: implications for dating young sediments. Quaternary Science Reviews (Quaternary Geochronology) 17: 1033-1040.

Rhodes EJ, 1990. The optical dating of quartz from sediments. Unpublished DPhil thesis, University of Oxford: $153 \mathrm{pp}$
Rhodes EJ, Bronk-Ramsey C, Outram Z, Batt C, Willis L, Dockrill S and Bond J, 2003. Bayesian methods applied to the interpretation of multiple OSL dates: high precision sediment age estimates from Old Scatness Broch excavations, Shetland Isles. Quaternary Science Reviews 22: 1231-1244.

Rhodes EJ and Pownall L, 1994. Zeroing of the OSL signal in quartz from young glaciofluvial sediments. Radiation Measurements 23 . 329-333.

Smith BW, Rhodes EJ, Stokes S, Spooner NA and Aitken MJ, 1990. Optical dating of sediments: initial results from Oxford. Archaeometry 32: 19-31.

Tijms H, 2004. Understanding Probability: Chance Rules in Everyday Life. Cambridge University Press, Cambridge: $378 \mathrm{pp}$.

Wallinga J, 2002. On the detection of OSL age overestimation using single-aliquot techniques. Geochronometria 21: 17-26. 